\title{
A Case of Unilateral Exophthalmos Due To Thyroid Orbitopathy in A Clinically Euthyroid Patient
}

\author{
Adeel Ahmad Khan, Ahmad Radwan Mahmoud Muammar, Ijaz Kamal
}

\begin{abstract}
Graves ophthalmopathy (GO) is the most common extra-thyroidal manifestation of Graves' disease (GD). Most cases of GO are bilateral whereas unilateral ophthalmopathy is less common. We report a case of a 24-year-old male with right sided exophthalmos for 6 months. He did not have any other visual or thyroid related symptoms. Laboratory results revealed low TSH, normal free $\mathrm{T} 3$ and free $\mathrm{T} 4$, elevated serum creatinine (418 umol/L) and positive TSH receptor antibodies US KUB showed features of chronic kidney disease (CKD). MRI Head showed unilateral thyroid associated orbitopathy. GO is still the most common cause of unilateral exophthalmos. Association between CKD and unilateral GO in clinically euthyroid patient is rare.
\end{abstract}

Index Terms - Chronic kidney disease (CKD), Glucocorticoids (GC), Graves' disease (GD), Graves ophthalmopathy (GO), Unilateral Exophthalmos

\section{INTRODUCTION}

Graves' disease (GD) is a disorder of autoimmune aetiology and Graves' ophthalmopathy (GO) is its most common extra-thyroidal manifestation (1). Though GO and hyperthyroidism of GD usually occur simultaneously, up to $5 \%$ of patients have been reported to be euthyroid at presentation (2)(3). GO is more common in females with annual incidence rate of 16 women and 3 men per million population (3). Most cases of GO are bilateral which may be asymmetric, whereas unilateral ophthalmopathy is less common (9-15\% cases) (4). TSH receptor auto-antibodies play an important role in the pathogenesis of $\mathrm{GO}$ as is evident by high expression of TSH receptor in orbital tissues. Increased volume of intra-orbital tissues is responsible for most of the symptoms of GO (5). Lid retraction, lid lag, exophthalmos, extra-ocular muscle involvement and decrease in vision are the most common manifestations of GO. Possible association between GD and primary kidney disease is rare and there are only few reported cases in literature (6). We report a case of unilateral exophthalmos in a euthyroid patient with positive TSH receptor antibodies with improvement in exophthalmos after steroid therapy. Furthermore, chronic kidney disease (CKD) was also observed in the patient.

Dr. Adeel Ahmad Khan, Medical Resident, Department of Internal Medicine, Hamad Medical Corporation, Doha, Qatar.

Dr. Ahmad Radwan Mahmoud Muammar, Medical Resident, Department of Internal Medicine, Kent Hospital/Brown University, USA.

Dr. Ijaz Kamal, Senior Consultant, Department of Internal Medicine, Hamad Medical Corporation, Doha, Qatar.

\section{CASE REPORT}

A 24-year-old male with no previous history of any chronic medical illnesses, presented to the clinic with protruded right eye for the past 6 months that was progressively getting worse. There was no eye pain, visual changes, ophthalmoplegia, dryness or discharge from eye. Patient did not report any fever, increased sweating, heat or cold intolerance, weight changes, appetite changes, headache, nausea or vomiting. He did not have any family history of thyroid diseases or orbital tumours and denied any history of smoking tobacco, drinking alcohol or using illicit drugs.

Physical examination revealed a comfortable and well-built young man with protruded right eye, lid retraction (Figure 1,2), normal eye movements and no signs of orbital cellulitis. Neck examination was significant for a mild diffuse goitre. Laboratory studies were significant for microcytic normochromic anaemia with haemoglobin of $12.1 \mathrm{~g} / \mathrm{dl}$ (normal $13-17 \mathrm{~g} / \mathrm{dl}$ ). He also had elevated serum creatinine at 418 umol/L (normal 64 - 110 umol/L). Serum electrolytes, liver function tests and lipid profile were within normal range. $24 \mathrm{hr}$ urine collection showed $3.08 \mathrm{gm} / 24 \mathrm{hr}$ proteinuria. Serum TSH was $0.04 \mathrm{mIU} / \mathrm{L}$ (normal $0.45-4.5$ $\mathrm{mIU} / \mathrm{L}$ ), free T4 was $13.8 \mathrm{pmol} / \mathrm{L}$ (normal $9-20 \mathrm{pmol} / \mathrm{L}$ ) and free T3 was $4.56 \mathrm{pmol} / \mathrm{L}$ (normal $2.89-4.88 \mathrm{pmol} / \mathrm{L}$ ). Thyrotropin Receptor Ab titre was 4.69 IU/L (normal 0.00 $1.75 \mathrm{IU} / \mathrm{L})$. ANA, ANCA, C3, C4, Anti thyroid peroxidase and Anti GBM antibodies were negative. Screening for hepatitis B, C and HIV was negative

US KUB showed bilateral small sized kidneys and increased parenchymal echogenicity suggestive of CKD. MRI Head was remarkable for proptosis of the right eye with increased retro-orbital fat, thickening and T2 hyper-intensity with sparing of the tendinous insertion involving the right inferior, medial, superior and lateral rectus muscles with crowding at the orbital apex. Features were suggestive of unilateral thyroid associated orbitopathy (Fig. 3). Patient received 1-week course of oral prednisolone $10 \mathrm{mg}$ per day after which his exophthalmos improved (Fig 4). An association between CKD and GO in a clinically euthyroid patient is rare.

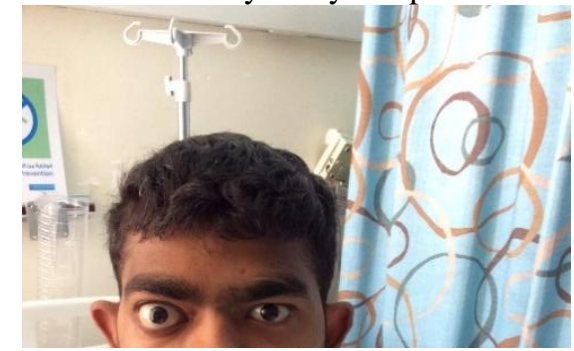

Fig.1 Right eye Exophthalmos 


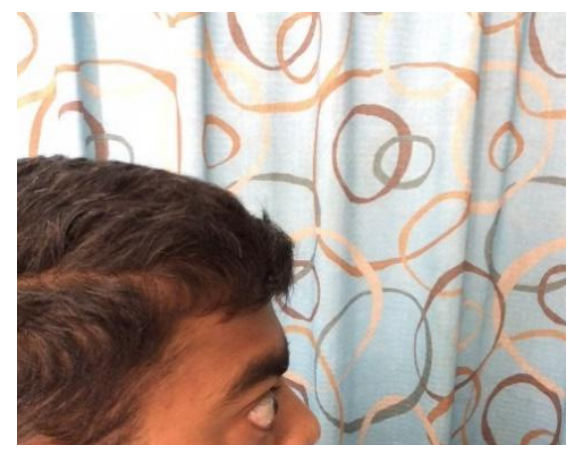

Fig. 2 Right eye Exophthalmos

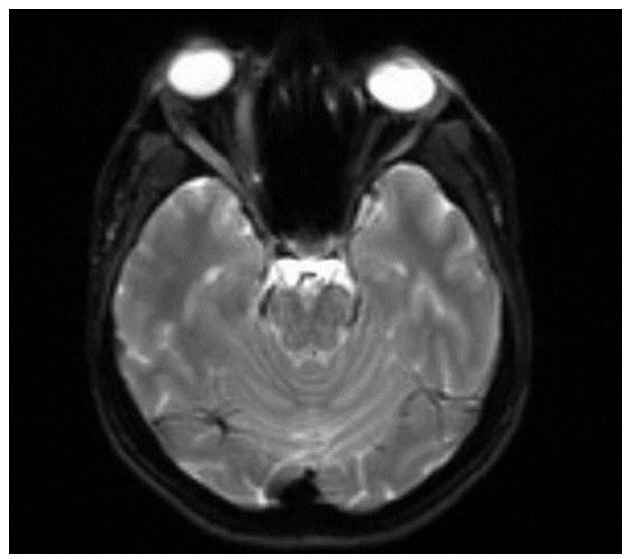

Fig. 3 Thickening of rectus muscles

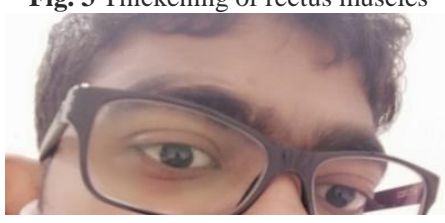

Fig. 4 After steroids treatment

\section{DISCUSSION}

GO usually presents simultaneously with GD. However, up to $5 \%$ patients with GO are euthyroid at presentation (2)(3). Both bilateral and unilateral eye involvement has been reported. However, in a euthyroid patient, presence of unilateral eye disease or exophthalmos presents a diagnostic dilemma leading to unnecessary and extensive testing on suspicion of orbital tumours. In a prospective study, up to $14 \%$ incidence of unilateral eye disease in GO has been observed (7). However, euthyroid patients are more likely to have unilateral eye involvement as compared to hyperthyroid individuals. Jang et al reported increased incidence of unilateral eye involvement $(79.2 \%)$ in euthyroid patients as compared to patients with hyperthyroidism (27.3\%) (8). Eckstein et al also reported increased incidence of asymmetric eye involvement in euthyroid patients as compared to hyperthyroid patients (9). Moreover, both studies noted milder and less severe clinical features in euthyroid patients with thyroid associated orbitopathy (8)(9). Glucocorticoids (GC) are the mainstay of treatment for moderate-severe GO defined as symptomatic GO but no evidence of sight threatening manifestations. IV GC are preferred over oral steroids. Second line options include orbital radiotherapy, cyclosporine, mycophenolate, rituximab and methotrexate (10).

Association between autoimmune thyroid diseases and CKD is rare. Tanwani et al reported a case of 29 years old female with GD and minimal change disease that responded well to treatment with radio-iodine and corticosteroids (11). Becker et al reported a case of membranous glomerulonephritis (GN) associated with GD. Patient developed kidney involvement after treatment with radio-iodine. (12). Keenswijk et al reported another case of GD with membranoproliferative glomerulonephritis (MPGN) in an 11 years old girl (13).

MPGN has been reported in other autoimmune thyroid disorders as well. Gurkan et al reported a case of 20 years old female who presented with symptoms of hypothyroidism and was diagnosed as autoimmune thyroiditis. Patient also had pitting edema and proteinuria at presentation and biopsy showed MPGN further validating association between thyroid disease and kidney involvement (14).

Our case report shows association between GO and CKD in a patient who is euthyroid. Although this case lacks biopsy evidence, presence of proteinuria along with positive TSH receptor antibodies raises suspicion of similar mechanism of association between thyroid disease and chronic kidney disease as reported in other such cases cited above.

\section{LEARNING POINTS}

- Unilateral exophthalmos presents a diagnostic dilemma especially in a euthyroid patient and exposes patients to unnecessary testing to rule out sinister causes. Graves' ophthalmopathy is still the most common cause in such cases.

- An association between GO in a euthyroid patient and $\mathrm{CKD}$ is a rare phenomenon and sometimes, kidney disease dominates the clinical picture.

\section{FUNDING STATEMENT}

This research did not receive any specific grant from any funding agency in the public, commercial or not-for-profit sector.

\section{DECLARATION OF INTEREST}

There is no conflict of interest that could be perceived as prejudicing the impartiality of the research reported.

\section{REFERENCES}

[1] "IMAJ $\mid$ The Israel Medicine Association Journal | Volume 19, Number 3, March 2017 | Graves' Opthalmopathy."

[2] Bahn, Rebecca S. "Graves' Ophthalmopathy." The New England Journal of Medicine 362, no. 8 (February 25, 2010): 726-38. https://doi.org/10.1056/NEJMra0905750.

[3] Bartley, G. B. "The Epidemiologic Characteristics and Clinical Course of Ophthalmopathy Associated with Autoimmune Thyroid Disease in Olmsted County, Minnesota." Transactions of the American Ophthalmological Society 92 (1994): 477-588.

[4] Kashkouli, Mohsen Bahmani, Reza Kaghazkanani, Iraj Heidari, Nooshin Ketabi, Sara Jam, Shahrzad Azarnia, and Farzad Pakdel. "Bilateral versus Unilateral Thyroid Eye Disease." Indian Journal of Ophthalmology 59, no. 5 (October 2011): 363-66. https://doi.org/10.4103/0301-4738.83612. 


\section{ISSN:2455-3956, Volume-9, Issue-6, December 2019 Pages 01-03}

[5] Bahn, Rebecca S. "Pathophysiology of Graves' Ophthalmopathy: The Cycle of Disease." The Journal of Clinical Endocrinology \& Metabolism 88, no. 5 (May 1, 2003): 1939-46. https://doi.org/10.1210/jc.2002-030010.

[6] Hasnain, Wirasat, Isaac E. Stillman, and George P. Bayliss. "Minimal-Change Renal Disease and Graves' Disease: A Case Report and Literature Review." NDT Plus 4, no. 2 (April 2011): 96-98. https://doi.org/10.1093/ndtplus/sfq213.

[7] Wiersinga WM, Smit T, van der Gaag R, Mourits M, Koornneef L. Clinical presentation of Graves' ophthalmopathy. Ophthalmic Res. 1989;21(2):73-82.

[8] Jang SY, Lee SY, Lee EJ, Yoon JS. Clinical features of thyroid-associated ophthalmopathy in clinically euthyroid Korean patients. Eye Lond Engl. 2012 Sep;26(9):1263-9.

[9] Eckstein AK, Lösch C, Glowacka D, Schott M, Mann K, Esser J, et al. Euthyroid and primarily hypothyroid patients develop milder and significantly more asymmetrical Graves ophthalmopathy. $\mathrm{Br} \mathrm{J}$ Ophthalmol. 2009 Aug;93(8):1052-6.

[10] Genere N, Stan MN. Current and Emerging Treatment Strategies for Graves' Orbitopathy. Drugs. 2019 Feb;79(2):109-24.

[11] Tanwani LK, Lohano V, Broadstone VL, Mokshagundam SPL. Minimal change nephropathy and graves' disease: report of a case and review of the literature. Endocr Pract Off J Am Coll Endocrinol Am Assoc Clin Endocrinol. 2002 Feb;8(1):40-3.

[12] Becker BA, Fenves AZ, Breslau NA. Membranous glomerulonephritis associated with Graves' disease. Am J Kidney Dis Off J Natl Kidney Found. 1999 Feb;33(2):369-73.

[13] Keenswijk W, Degraeuwe E, Hoorens A, Dorpe JV, Vande Walle J. A case of Graves' disease associated with membranoproliferative glomerulonephritis and leukocytoclastic vasculitis. J Pediatr Endocrinol Metab JPEM. 2018 Oct 25;31(10):1165-8.

[14] Gurkan S, Dikman S, Saland MJ. A case of autoimmune thyroiditis and membranoproliferative glomerulonephritis. Pediatr Nephrol Berl Ger. 2009 Jan;24(1):193-7.

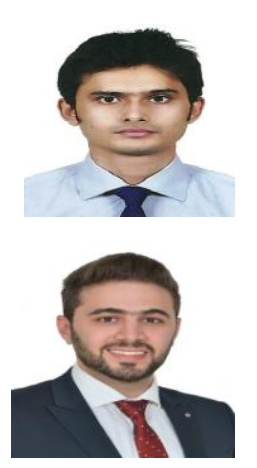

Dr Adeel Ahmad Khan, MBBS (Nishtar Medical College, Multan, Pakistan) is $3^{\text {rd }}$ Year Internal Medicine Resident in Hamad Medical Corporation, Doha, Qatar.

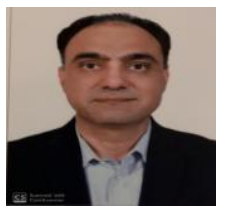

Dr. Ijaz Kamal (MBBS, MRCPI, FRCPI) is consultant physician in department of Internal Medicine at Hamad General Hospital, Doha, Qatar And Assistant Professor of Clinical Medicine at Weill Cornell Medical School, Qatar. He has CCST in General Internal Medicine\& Acute medicine by Royal College of Physicians Ireland (RCPI) with Special interest in Respiratory Medicine. 